\title{
GIBBS PHENOMENON AND CERTAIN NONHARMONIC FOURIER SERIES
}

\author{
JungSOO RHEE
}

\begin{abstract}
The Fourier series has a rapid oscillation near end points at jump discontinuity which is called the Gibbs phenomenon. There is an overshoot (or undershoot) of approximately $9 \%$ at jump discontinuity.

In this paper, we prove that a bunch of series representations (certain nonharmonic Fourier series) give good approximations vanishing Gibbs phenomenon. Also we have an application for approximating some shape of upper part of a vehicle in a different way from the method of cubic splines and wavelets.
\end{abstract}

\section{Introduction}

The Fourier series of the function $f$ is an infinite series:

$$
a_{0}+\sum_{n=0}^{\infty}\left(a_{n} \cos \frac{n \pi x}{L}+b_{n} \sin \frac{n \pi x}{L}\right),
$$

where the Fourier coefficients of $f$ are defined by $a_{0}=\frac{1}{2 L} \int_{-L}^{L} f(x) d x, a_{n}=$ $\frac{1}{L} \int_{-L}^{L} f(x) \cos \frac{n \pi x}{L} d x$, and $b_{n}=\frac{1}{L} \int_{-L}^{L} f(x) \sin \frac{n \pi x}{L} d x, n \in \mathbb{N}$, where $\mathbb{N}$ denotes the set of positive integers.

To check the convergence of Fourier series, we consider the partial sum of the first $2 N+1$ terms of the series,

$$
S_{N}(x)=a_{0}+\sum_{n=0}^{N}\left(a_{n} \cos \frac{n \pi x}{L}+b_{n} \sin \frac{n \pi x}{L}\right) .
$$

Also $S_{N}$ can be noted by convolution with the Dirichlet kernel $D_{N}$ :

$$
S_{N}(x)=f * D_{N}(x),
$$

where $D_{N}$ is given by $D_{N}(x)=\frac{1}{2}+\sum_{n=1}^{N} \cos \frac{n \pi}{L} x$. The Dirichlet kernel $D_{N}$ plays a very important role to make Gibbs phenomenon of the Fourier series

Received January 15, 2010.

2010 Mathematics Subject Classification. 42A20, 42A24.

Key words and phrases. Gibbs phenomenon, certain nonharmonic Fourier series, splines and wavelets. 
at the jump discontinuity of the step function $H$ defined by

$$
H(x)= \begin{cases}0, & \text { if } \quad-1<x<0 \\ 1, & \text { if } \quad 0 \leq x<1 .\end{cases}
$$

Hence if we modify $D_{N}$ properly, we might get rid of jumps at the end points.

Before getting into the main subject, let's have a typical example for Gibbs phenomenon. Since $H$ is a step function, we get the Fourier series $S(x)=$ $\frac{1}{2}+\sum_{n=1}^{\infty} \frac{1-(-1)^{n}}{n \pi} \sin n \pi x$. As seen in Figure 2.1, the graph of the partial sum $S_{30}$ has spikes near the point of discontinuity, at $x=0$. One can refer to [4] and [8] for detailed Gibbs phenomenon.

In the next section, we will replace $D_{N}$ by $\tilde{K}_{m, n}$ to smooth out these overshooting.

\section{Main results}

We are ready to develop a certain convolution generating a bunch of nonharmonic Fourier series that vanishes the Gibbs phenomenon. We consider kernels

$$
\tilde{K}_{n}(x)=n \int_{0}^{1} g(t) \cos 2 \pi n t x d t .
$$

Then the following theorem holds.

Theorem 2.1. Suppose that $g$ is continuous on $[0,1]$, and suppose that $\tilde{K}_{1} \in$ $L^{1}(\mathbb{R})$ with $\int_{\mathbb{R}} \tilde{K}_{1}(x) d x=a$, where $\mathbb{R}$ denotes the set of real numbers.

(a) Suppose that $g^{\prime}, g^{\prime \prime}$ are continuous on $[0,1]$ and $g(1)=0$. If $f \in L^{1}(\mathbb{R})$, we have $\lim _{n \rightarrow \infty} f * \tilde{K}_{n}(\theta)=$ af $(\theta)$ for almost all $\theta \in \mathbb{R}$.

(b) If $f \in L^{p}(\mathbb{R})$, then $f * \tilde{K}_{n} \rightarrow$ af in the $L^{p}$ norm as $n \rightarrow \infty$ for $p \geq 1$.

(c) If $f$ is bounded and uniformly continuous, then $f * \tilde{K}_{n} \rightarrow$ af uniformly as $n \rightarrow \infty$.

(d) If $f \in L^{\infty}(\mathbb{R})$ and $f$ is continuous on an open set $U$, then $f * \tilde{K}_{n} \rightarrow$ af uniformly on compact subsets of $U$.

Proof. We will copy the typical proof of this type of convergence theorem from [3] and [7]. First, we show that $\left|f * \tilde{K}_{n}(\theta)\right| \leq M f(\theta)$ for almost all $\theta \in \mathbb{R}$ and $n \in \mathbb{N}$, where $M f$ is the Hardy-Littlewood Maximal function, i.e., $M f(\theta)=$ $\sup _{x>0} \frac{1}{x} \int_{-x}^{x}|f(\theta-t)| d t$.

More precisely, we calculate as follows:

$$
\begin{aligned}
f * \tilde{K}_{n}(\theta) \leq & \int_{|y|<\frac{1}{n}}|f(\theta-y)| n \int_{0}^{1}|g(t) \cos 2 \pi n t y| d t d y \\
& +\left|\int_{|y| \geq \frac{1}{n}} f(\theta-y) \tilde{K}_{n}(y) d y\right| \\
= & I_{1}+I_{2} .
\end{aligned}
$$

By the definition of the maximal function, we get $\left|I_{1}\right| \leq\|g\|_{\infty} M f(\theta)$. 
Integration by parts tells us that $\left|\tilde{K}_{n}(x)\right| \leq \frac{c}{n x^{2}}$, when $x \neq 0$ and $c=$ $\frac{2\left\|g^{\prime}\right\|_{\infty}+\left\|g^{\prime \prime}\right\|_{\infty}}{4 \pi^{2}}$. Hence we have

$$
\begin{aligned}
I_{2} & \leq c \int_{\frac{1}{n}}^{\infty}|f(\theta-x)+f(\theta+x)| \frac{1}{n x^{2}} d x \\
& =I_{3} .
\end{aligned}
$$

Set $F_{\theta}(x)=\int_{0}^{x}|f(\theta-t)+f(\theta+t)| d t$. Then we obtain, by integration by parts,

$$
\begin{aligned}
I_{3} & \leq \frac{c}{n}\left|\left[F_{\theta}(x) x^{-2}\right]_{x=\frac{1}{n}}^{\infty}\right|+\frac{2 c}{n} \int_{\frac{1}{n}}^{\infty} F_{\theta}(x) x^{-3} d x \\
& \leq 3 c M f(\theta) .
\end{aligned}
$$

Since $\operatorname{Mf}(\theta)$ is finite for almost all $\theta \in \mathbb{R}, \lim _{n \rightarrow \infty} f * \tilde{K}_{n}(\theta)$ exist for almost all $\theta \in \mathbb{R}$.

The definition of the convolution shows that

$$
f * \tilde{K}_{n}(\theta)-a f(\theta)=\int_{\mathbb{R}}\left[f\left(\theta-\frac{x}{n}\right)-f(\theta)\right] \tilde{K}_{1}(x) d x .
$$

Since $\tilde{K}_{1}(x) \in L^{1}(\mathbb{R}), f * \tilde{K}_{n} \rightarrow$ af in the $L^{p}$ norm as $n \rightarrow \infty$ for $p \geq 1$ by the Minkowski's inequality for integrals and the dominated convergence theorem. This proves (a), by the consistence of mean and pointwise convergence, and (b).

Hence, it is natural to think about whether the above convergence is uniform or not, where $f$ is in a good condition. Actually, $\left\|f\left(\cdot-\frac{x}{n}\right)-f(\cdot)\right\|_{\infty}$ tends to zero uniformly as $n \rightarrow \infty$ for all $x \in K$, any compact subset of $\mathbb{R}$, by the uniform continuity of $f$ in case $(\mathrm{c})$.

Hence we have

$$
\begin{aligned}
\left\|f * \tilde{K}_{n}-a f\right\|_{\infty} \leq & \int_{K}\left\|f\left(\cdot-\frac{x}{n}\right)-f(\cdot)\right\|_{\infty}\left|\tilde{K}_{1}(x)\right| d x \\
& +\int_{K^{c}} 2\|f\|_{\infty}\left|\tilde{K}_{1}(x)\right| d x .
\end{aligned}
$$

If we choose $K$ such that $\int_{K^{c}}\left|\tilde{K}_{1}(x)\right| d x<\epsilon$, the desired result for (c) follows from the boundedness of $f$ and the dominated convergence theorem. Therefore, this gives our proof of (c).

As for (d), given $\epsilon>0$, choose a compact subset $E \subset \mathbb{R}$ such that $\int_{E^{c}}\left|\tilde{K}_{1}(x)\right| d x$ $<\epsilon$. Also we let $F$ be a compact subset of $U$. If $\theta \in F$ and $x \in E$, then we will have $\theta-\frac{x}{n} \in U$ for sufficiently large $n$. From the compactness of $F$, it follows that

$$
\sup _{\theta \in F, x \in E}\left|f\left(\theta-\frac{x}{n}\right)-f(\theta)\right|<\epsilon
$$

for large $n$.

In fact, for each $\theta \in F$ there exist $\delta_{\theta}>0$ such that $\left|f\left(\theta-\frac{x}{n}\right)-f(\theta)\right|<\frac{\epsilon}{2}$ if $\left|\frac{x}{n}\right|<\delta_{\theta}$. Since $F$ is compact, there exist $\theta_{1}, \ldots, \theta_{N}$ such that the balls of radius 
$\delta_{\theta_{i}}, i=1,2, \ldots, N$, cover $F$. Now we let $\delta=\frac{1}{2} \min \delta_{\theta_{i}}$. Then we conclude that $\left|f\left(\theta-\frac{x}{n}\right)-f(\theta)\right|<\epsilon$ for each $\theta \in F$ and $x \in E$, whenever $\left|\frac{x}{n}\right|<\delta$.

Hence we obtain

$$
\begin{aligned}
\sup _{\theta \in F}\left|f * \tilde{K}_{n}(\theta)-a f(\theta)\right| & \leq \sup _{\theta \in F}\left[\int_{E}+\int_{E^{c}}\right]\left|f\left(\theta-\frac{x}{n}\right)-f(\theta)\right|\left|\tilde{K}_{1}(x)\right| d x \\
& \leq \epsilon \int_{\mathbb{R}}\left|\tilde{K}_{1}(x)\right| d x+2 \epsilon\|f\|_{\infty} .
\end{aligned}
$$

This completes the proof of $(d)$.

As we already have mentioned, we need a slight modification of the Dirichlet kernel $D_{n}$ in order to vanish the Gibbs phenomenon at the point of jump discontinuity. We will construct certain kernel $\tilde{K}_{m, n}$ that converges to $\tilde{K}_{n}$. It might have many ways to define these partial sum $\tilde{K}_{m, n}$. Since $\tilde{K}_{n}$ is a dilation of a Fourier transform, these partial sums $\tilde{K}_{m, n}$ can be nonharmonic Fourier series.

To make problem simple, we define $\tilde{K}_{m, n}$ by a Riemann sum of $\tilde{K}_{n}$ using the trapezoidal rule, i.e.,

$$
\tilde{K}_{m, n}(x)=\frac{n}{m}\left[\frac{g(0)}{2}+\sum_{k=1}^{m-1} g\left(\frac{k}{m}\right) \cos 2 \pi n \frac{k}{m} x\right],
$$

when $g(1)=0$.

Now we will show that these discretizations $\tilde{K}_{m, n}$ of kernel $\tilde{K}_{n}$ guarantee the uniform convergence with proper conditions:

Theorem 2.2. Suppose that $g$ is a continuous function on $[0,1]$. If $f \in C_{c}(\mathbb{R})$, then $f * \tilde{K}_{m, n} \rightarrow f * \tilde{K}_{n}$ uniformly on any compact subset of $\mathbb{R}$ as $m \rightarrow \infty$ for fixed nonnegative integer $n$.

Proof. Let $E$ be any compact subset of $\mathbb{R}$. Since $f$ has a compact support, we have some interval $[-M, M]$ for large $M>0$, satisfying the following inequality:

$$
\left|f * \tilde{K}_{m, n}(\theta)-f * \tilde{K}_{n}(\theta)\right| \leq \int_{-M}^{M}|f(\theta-y)|\left|\tilde{K}_{m, n}(y)-\tilde{K}_{n}(y)\right| d y
$$

for all $\theta \in E$. Therefore it is enough to show that $\left|\tilde{K}_{m, n}(y)-\tilde{K}_{n}(y)\right|$ converges to zero uniformly on $[-M, M]$ as $m \rightarrow \infty$.

Let $\epsilon>0$ be given. A direct calculation gives us

$$
\begin{aligned}
& \left|\tilde{K}_{n}(y)-\tilde{K}_{m, n}(y)\right| \\
\leq & \left(\frac{n}{m} \frac{|g(0)|}{2}+\sum_{k=0}^{m-1} \int_{\frac{k}{m}}^{\frac{k+1}{m}} n|g(t)|\left|\cos 2 \pi n t y-\cos 2 \pi n \frac{k}{m} y\right| d t\right)
\end{aligned}
$$




$$
\begin{aligned}
& +\sum_{k=0}^{m-1} \int_{\frac{k}{m}}^{\frac{k+1}{m}} n\left|g(t)-g\left(\frac{k}{m}\right)\right| d t \\
= & I_{1}+I_{2} .
\end{aligned}
$$

Since $g$ is uniformly continuous on $[0,1]$, there exists $\delta>0$ such that $\mid g(x)-$ $g(y) \mid<\frac{\epsilon}{n}$ whenever $|x-y|<\delta$. Thus, we can choose a positive integer $L$ such that $\frac{1}{m}<\delta$ for any $m \geq L$. Then we have $\left|g(t)-g\left(\frac{k}{m}\right)\right|<\frac{\epsilon}{n}$ for all $t \in\left[\frac{k}{m}, \frac{k+1}{m}\right]$ and for each $k=0,1, \ldots, m-1$. Therefore $I_{2} \leq \epsilon$ for fixed $n$ whenever $m \geq L$.

For $I_{1}$, we have $\left|\cos 2 \pi n t y-\cos 2 \pi n \frac{k}{m} y\right| \leq|2 \pi n y|\left|2 \pi n t y-2 \pi n \frac{k}{m} y\right|$ by the mean value theorem for each $t \in[0,1]$. Since $g$ is bounded on $[0,1]$ and $y \in[-M, M]$, we get

$$
\begin{aligned}
I_{1} & \leq \frac{n}{m} \frac{|g(0)|}{2}+\sum_{k=0}^{m-1} \int_{\frac{k}{m}}^{\frac{k+1}{m}} n|g(t)|(2 \pi n y)^{2}\left(t-\frac{k}{m}\right) d t \\
& \leq \frac{n}{m} \frac{|g(0)|}{2}+\frac{1}{m}\left(2 \pi^{2} n^{3} M^{2}\|g\|_{\infty}\right) \leq \epsilon
\end{aligned}
$$

for large $m$.

Hence we can conclude

$$
\left|f * \tilde{K}_{m, n}(\theta)-f * \tilde{K}_{n}(\theta)\right| \leq 2 \epsilon\|f\|_{1}
$$

for large $m$ and for all $\theta \in E$. This completes our theorem.

From the definition of $\tilde{K}_{m, n}$, we can induce a nonharmonic Fourier series. For $f \in L^{1}(\mathbb{R})$, we have

$$
\begin{aligned}
& f * \tilde{k}_{m, n}(x) \\
= & \frac{n}{m}\left[\frac{g(0)}{2} a_{n, m}^{0}+\sum_{k=1}^{m-1} g\left(\frac{k}{m}\right)\left(a_{n, m}^{k} \cos 2 \pi n \frac{k}{m} x+b_{n, m}^{k} \sin 2 \pi n \frac{k}{m} x\right)\right],
\end{aligned}
$$

where $a_{n, m}^{k}=\int_{\mathbb{R}} f(y) \cos 2 \pi n \frac{k}{m} y d y$ and $b_{n, m}^{k}=\int_{\mathbb{R}} f(y) \sin 2 \pi n \frac{k}{m} y d y$.

Actually $f * \tilde{K}_{m, n}$ converges to af uniformly when $f$ is in a good condition. Hence $H * \tilde{K}_{m, n}$ might get rid of the Gibbs phenomenon for the step function $H$.

Theorem 2.3. (a) If $f \in C_{c}(\mathbb{R})$, then

$$
\lim _{n \rightarrow \infty} \lim _{m \rightarrow \infty} f * \tilde{K}_{m, n}(\theta)=a f(\theta),
$$

uniformly for all $\theta \in E$, where $E$ is any compact subset of $\mathbb{R}$ and $a$ is the same constant in Theorem 2.1.

(b) Suppose that $g(0)=2, g(1)=0$, and that $g$ and $g^{\prime}$ are continuous on $[0,1]$. If $g$ is decreasing and $\left\|g^{\prime}\right\|_{\infty} \leq 2$ on $[0,1], H * \tilde{K}_{m, n}$ do not show up the Gibbs phenomenon on $(-1,1)$ at the point of jump discontinuity (i.e., at $x=0)$. 
Proof. Combining Theorem 2.1 and Theorem 2.2, (a) is easily proven. We will prove (b) by a direct calculation. As in the proof of Theorem 2.2, we prove that

$$
\left|\tilde{K}_{m, n}(y)-\tilde{K}_{n}(y)\right| \leq \epsilon
$$

for any $y \in[-M, M]$ and for $m$ large enough and some $M>0$. Thus we have

$$
\begin{aligned}
H * K_{m, n}(\theta) & \leq \int_{0}^{1}\left|\tilde{K}_{m, n}(\theta-y)-\tilde{K}_{n}(\theta-y)\right| d y+\left|\int_{0}^{1} \tilde{K}_{n}(\theta-y) d y\right| \\
& <\epsilon+\left|I_{n}(\theta)\right|
\end{aligned}
$$

for large $m$ and for all $\theta \in(-1,1)$, when we put $I_{n}(\theta)=\int_{0}^{1} \tilde{K}_{n}(\theta-y) d y$.

Now we are going to check the Gibbs phenomenon near the jump discontinuity at $x=0$. From the above inequality and the definition for $H * \tilde{K}_{m, n}$, we have

$$
\lim _{n \rightarrow \infty} \lim _{m \rightarrow \infty} H * \tilde{K}_{m, n}(\theta)=\lim _{n \rightarrow \infty} I_{n}(\theta) .
$$

Then $I_{n}$ converges to 0 for $-1<\theta<0,1$ for $0<\theta<1$, and $\frac{1}{2}$ for $\theta=0$, from a simple calculation of $I_{n}$. In fact, we obtain

$$
\begin{aligned}
I_{n}(\theta) & =\int_{0}^{1} \int_{0}^{1} g(t) n \cos 2 \pi n(\theta-y) t d t d y \\
& =-\int_{0}^{1} g^{\prime}(t) \int_{n(\theta-1) t}^{n \theta t} \frac{\sin 2 \pi z}{2 \pi z} d z d t .
\end{aligned}
$$

Hence, if we take $m \rightarrow \infty$ followed by $n \rightarrow \infty$, we obtain $H * \tilde{K}_{m, n}(\theta)$ converges to 0 for $-1<\theta<0,1$ for $0<\theta<1$, and $\frac{1}{2}$, the average of $H(0+)$ and $H(0-)$, for $\theta=0$.

In order to check spikes near at $x=0$, we need a more detailed calculation of $I_{n}$ for $\theta>0$. When we put $F(r)=\int_{0}^{r} \frac{\sin x}{x} d x$ as the sine integral, we obtain

$$
\begin{aligned}
I_{n}(\theta) & =\frac{1}{2 \pi} \int_{0}^{1}\left(-g^{\prime}(t)\right)[F(2 \pi n \theta t)+F(2 \pi n(1-\theta) t)] d t \\
& \leq \frac{1}{2 \pi}\left\|g^{\prime}\right\|_{\infty}\left(\frac{1}{2 \pi n \theta} \int_{0}^{2 \pi n \theta} F(r) d r+\frac{1}{2 \pi n(1-\theta)} \int_{0}^{2 \pi n(1-\theta)} F(r) d r\right) \\
& \leq \frac{\left\|g^{\prime}\right\|_{\infty}}{2} \frac{1}{\pi}\left(\int_{0}^{2 \pi n \theta} \frac{2 \sin ^{2} \frac{x}{2}}{x^{2}} d x+\int_{0}^{2 \pi n(1-\theta)} \frac{2 \sin ^{2} \frac{x}{2}}{x^{2}} d x\right) \\
& <\frac{\left\|g^{\prime}\right\|_{\infty}}{2} .
\end{aligned}
$$

Thus, if $\left\|g^{\prime}\right\|_{\infty} \leq 2$, we have $I_{n}(\theta)<1$ for all $0<\theta<1$ and for all $n$. By the exact copy of the proof in Theorem 2.2, we have $H * \tilde{K}_{m, n}(\theta) \leq O\left(\frac{1}{n}\right)+I_{n}(\theta)$ for $m=n^{4}$. Hence $H * \tilde{K}_{m, n}$ don't have any overshoot near the jump discontinuity at $\theta=0$, provided $m$ sufficiently large.

Also, we have 


$$
I_{n}(\theta)+I_{n}(-\theta)=\int_{0}^{1} \int_{n(\theta-1) t}^{n(\theta+1) t}\left(-g^{\prime}(t)\right) \frac{\sin 2 \pi x}{2 \pi x} d x d t .
$$

Thus we have $\lim _{n \rightarrow \infty}\left(I_{n}(\theta)+I_{n}(-\theta)\right)=1$ for all $0<\theta<1$. By this duality, $I_{n}(-\theta)$ has no undershoot near the jump discontinuity at $\theta=0$, whenever $0<\theta<1$. Therefore, $H * \tilde{K}_{m, n}$ don't have any undershoot for sufficiently large $m$. This completes the proof of (b).

\section{Applications}

In the previous section, we introduced some interesting kernel $\tilde{K}_{m, n}$ vanishing the Gibbs phenomenon. To get more specific examples, we let $g(t)=2(1-t)$ and we put $K_{n}=\int_{0}^{1} 2 n(1-t) \cos 2 \pi n t x d t$. Then we obtain a kernel

$$
K_{m, n}(x)=\frac{n}{m}\left[1+2 \sum_{k=1}^{m-1}\left(1-\frac{k}{m}\right) \cos 2 \pi n \frac{k}{m} x\right],
$$

by the same process of making $\tilde{K}_{m, n}$, that is a reminiscent of the Fejer kernel, $K_{n}(x)=\frac{\sin ^{2} \pi n x}{\pi^{2} n x^{2}}$ by a simple calculation of $K_{n}$.

By Theorem 2.1, Theorem 2.2 and Theorem 2.3, we know that $f * K_{n}$ and $f * K_{m, n}$ converges to $f$ uniformly on a compact space $K$ where it is continuous. Also Theorem 2.2 and Theorem 2.3 tell us that $H * K_{m, n}$ has no Gibbs phenomenon, for $m=n^{4}$, as $n \rightarrow \infty$.

But we obtain a sharper estimation by a direct calculation. In fact, since we have

$$
\begin{aligned}
H * K_{m, n}(x) & =\int_{0}^{1}\left(K_{m, n}(x-t)-K_{n}(x-t)\right) d t+\int_{0}^{1} K_{n}(x-t) d t \\
& =I_{1}+I_{2} .
\end{aligned}
$$

We obtain

$$
I_{1} \leq 2 n \int_{0}^{1}\left|1-\frac{\sin \pi \frac{n}{m}(x-t)}{\pi \frac{n}{m}(x-t)}\right| d t \leq O(1 / n),
$$

when $m=n^{2}$ and $x \in(-1,1)$. For details, see Lemma 2.1 in [6].

Also we have

$$
\begin{aligned}
I_{2} & =\int_{0}^{1} \frac{\sin ^{2} \pi n(x-t)}{\pi^{2} n(x-t)^{2}} d t \\
& =\frac{1}{\pi} \int_{\pi n(x-1)}^{\pi n x} \frac{\sin ^{2} s}{s^{2}} d s .
\end{aligned}
$$

Thus $H * K_{n^{2}, n}(x)$ converges to 0 for $-1<x<0$ and $1=\frac{1}{\pi} \int_{-\infty}^{\infty} \frac{\sin ^{2} s}{s^{2}} d s$ for $x>0$. For $x=0, H * K_{n^{2}, n}(x)$ converges to $1 / 2$, the average of $H(0+)$ and $H(0-)$, as we have expected.

Actually, we have $H * K_{n^{2}, n}(x)=O(1 / n)+\frac{1}{\pi} \int_{\pi n(x-1)}^{\pi n x} \frac{\sin ^{2} s}{s^{2}} d s \leq \epsilon+1$ for large $n$ and $0<x$. Hence we conclude that there is no overshooting when $0<x$. 
We also have $I_{1}+I_{2} \geq 0$ by Lemma 2.1 in [6]. Thus there is no undershooting when $x<0$. Therefore, we don't have any spike near $x=0$ at the jump discontinuity.

By a direct calculation of $H * K_{n^{2}, n}$, we have

$$
H * K_{n^{2}, n}(x)=\frac{1}{n}\left[1+2 \sum_{k=1}^{n^{2}-1}\left(1-\frac{k}{n^{2}}\right)\left(a_{n, k} \cos 2 \pi \frac{k}{n} x+b_{n, k} \sin 2 \pi \frac{k}{n} x\right)\right]
$$

where $a_{n, k}=\frac{\sin 2 \pi \frac{k}{n}}{2 \pi \frac{k}{n}}$, and $b_{n, k}=\frac{1-\cos 2 \pi \frac{k}{n}}{2 \pi \frac{k}{n}}$.

See the graph of $S_{n}=H * D_{n}, n=30$, on Figure 2.1 and the graph of $H * K_{n^{2}, n}, n=15$, on Figure 2.2. By the direct calculation that we have performed, the kernels $K_{n^{2}, n}$ don't give Gibbs phenomenon compared to the kernel $D_{n}$. We can also see the duality graphically on Figure 2.2, which has been mentioned in the proof of Theorem 2.3.

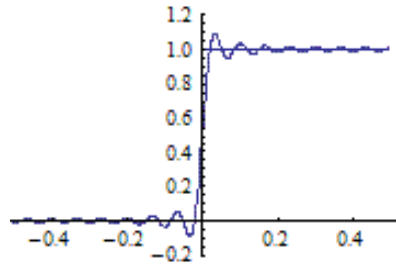

Figure 2.1. The graph of $S_{30}$

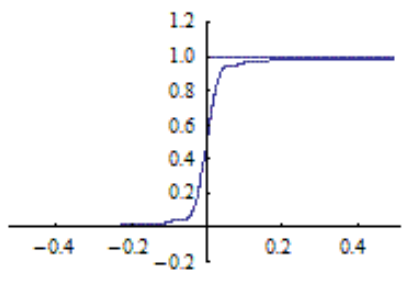

Figure 2.2. The graph of $\mathrm{f} * K_{15}{ }^{2}=15$

We take one more example to approximate an upper part of vehicle. Now we choose 9 points that form outlines of the vehicle: $(0.4,1.1),(0.6,1.1),(0.6,1.6)$, $(2,1.6),(3,2.5),(5.5,2.5),(5.8,2.1),(5.9,1.1),(6.2,1.1)$.

Then we can see the frame of this vehicle on Figure 2.3, that looks like the frame of Soul manufactured by Kia Automobile Company. Let $f$ be the graph of broken lines passing through these points. Of course, this function is not continuous at $x=0.6$ (i.e., the point of jump discontinuity). Actually $f$ is a piecewise continuous function. As an example, we have $f(x)=0.9 x-0.2$ on $[2,3)$.

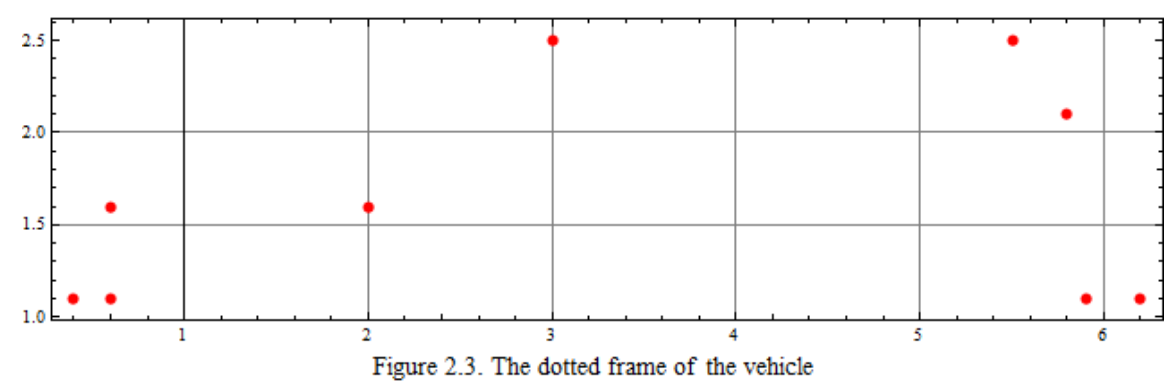


Now we will calculate $f * K_{m, n}$ directly. We obtain

$$
\begin{aligned}
& f * K_{m, n}(x) \\
= & \frac{n}{m}\left[a_{n, m}^{0}+2 \sum_{k=1}^{m-1}\left(1-\frac{k}{m}\right)\left(a_{n, m}^{k} \cos 2 \pi n \frac{k}{m} x+b_{n, m}^{k} \sin 2 \pi n \frac{k}{m} x\right)\right],
\end{aligned}
$$

when we get

$$
\begin{aligned}
a_{n, m}^{k}= & \int_{0.4}^{0.6} 1.1 \cos 2 \pi n \frac{k}{m} t d t+\int_{0.6}^{2} 1.6 \cos 2 \pi n \frac{k}{m} t d t \\
& +\int_{2}^{3}(0.9 x-0.2) \cos 2 \pi n \frac{k}{m} t d t+\int_{3}^{5.5} 2.5 \cos 2 \pi n \frac{k}{m} t d t \\
& +\int_{5.5}^{5.8}\left(-\frac{4}{3} x+\frac{59}{6}\right) \cos 2 \pi n \frac{k}{m} t d t \\
& +\int_{5.8}^{5.9}(-10 x+60.1) \cos 2 \pi n \frac{k}{m} t d t \\
& +\int_{5.9}^{6.2} 1.1 \cos 2 \pi n \frac{k}{m} t d t, k=0,1,2, \ldots, m-1
\end{aligned}
$$

and

$$
\begin{aligned}
b_{n, m}^{k}= & \int_{0.4}^{0.6} 1.1 \sin 2 \pi n \frac{k}{m} t d t+\int_{0.6}^{2} 1.6 \sin 2 \pi n \frac{k}{m} t d t \\
& +\int_{2}^{3}(0.9 x-0.2) \sin 2 \pi n \frac{k}{m} t d t+\int_{3}^{5.5} 2.5 \sin 2 \pi n \frac{k}{m} t d t \\
& +\int_{5.5}^{5.8}\left(-\frac{4}{3} x+\frac{59}{6}\right) \sin 2 \pi n \frac{k}{m} t d t \\
& +\int_{5.8}^{5.9}(-10 x+60.1) \sin 2 \pi n \frac{k}{m} t d t \\
& +\int_{5.9}^{6.2} 1.1 \sin 2 \pi n \frac{k}{m} t d t, k=1,2, \ldots, m-1 .
\end{aligned}
$$

As we have seen the graph of $f * K_{m, n}$ on Figure 2.4, $f * K_{m, n}$ converges to $f$ without showing Gibbs phenomenon. The convergence depends on how efficiently we take the number of data $m$ and $n$. In this occasion, we took $n=100, m=10 n$.

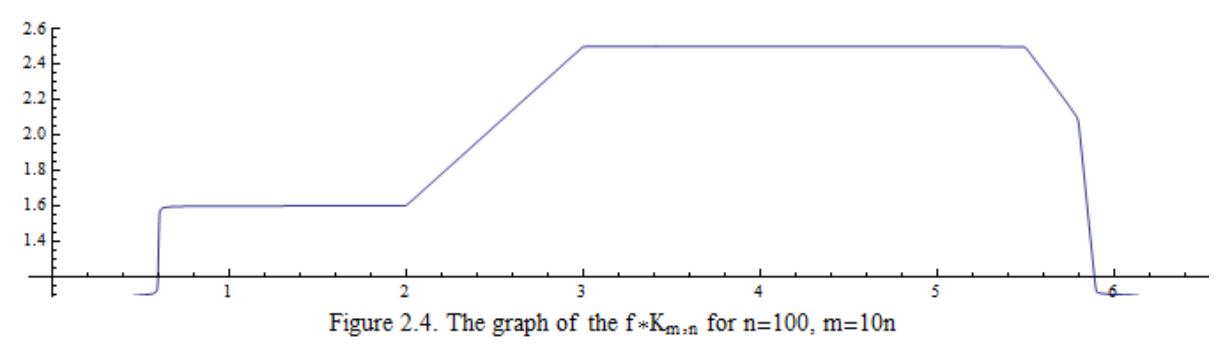


Of course, we can consider approximations of cubic splines or wavelets in comparison with our results. See [1], [2] and [5]. Also we notify that all pictures in this paper have been rendered by the help of Mathematica Version 7.0.

\section{References}

[1] C. K. Chui, An Introduction to Wavelets, Academic Press, 1992.

[2] I. Daubechies, Ten Lectures on Wavelets, CBMS-NSF Regional Conference Series in Applied Mathematics, Vol. 61, Society for Industrial and Applied Mathematics, Philadelphia, PA, 1992.

[3] G. B. Folland, Real Analysis, John Wiley \& Sons, Inc., New York, 1984.

[4] R. Haberman, Elementary Applied Partial Differential Equations, Prentice-Hall, Inc., Englewood Cliffs, N.J., 1983.

[5] T. H. Koornwinder, Wavelets: An Elementary Treatment of Theory and Applications, Series in Approximations and Decompositions-Vol.1, World Scientific, Singapole, 1995.

[6] J. Rhee, A nonharmonic Fourier series and dyadic subdivision schemes, East Asian Mathematical Journal 26 (2010), no. 1, 79-87.

[7] E. M. Stein, Harmonic Analysis: Real-Variable Methods, Orthogonality, and Oscillatory Integrals, Princeton University Press, Princeton, NJ, 1993.

[8] Antoni Zygmund, Trigonometric Series. Vol. I, II, Cambridge University Press, Cambridge-New York-Melbourne, 1977.

Department of Mathematics

Pusan University of Foreign Studies

PusAn 608-738, KoreA

E-mail address: rhee@pufs.ac.kr 